Thomas Richter - Die Dialoge über Literatur im Briefwechsel zwischen Goethe und Zelter 


\author{
Thomas Richter
}

\title{
Die Dialoge über Literatur im Briefwechsel zwischen Goethe und Zelter
}

Verlag J. B. Metzler Stuttgart - Weimar 
Die Deutsche Bibliothek - CIP-Einheitsaufnahme

Stuttgart ; Weimar : Metzler, 2000

ISBN 978-3-476-45230-6

ISBN 978-3-476-45230-6

ISBN 978-3-476-04322-1 (eBook)

DOI 10.1007/978-3-476-04322-1

Dieses Werk ist einschließlich aller seiner Teile geschützt. Jede Verwertung außerhalb der engen Grenzen des Urheberrechtsgesetzes ist ohne Zustimmung des Verlages unzulässig und strafbar. Das gilt insbesondere für die Vervielfältigungen, Übersetzung, Mikroverfilmungen und Einspeicherung in elektronischen Systemen.

(M-\&-P-Schriftenreihe für Wissenschaft und Forschung)

(C) 2000 Springer-Verlag GmbH Deutschland Ursprünglich erschienen bei J.B. Metzlersche Verlagsbuchhandlung und Carl Ernst Poeschel Verlag GmbH in Stuttgart 2000 


\section{Inhalt}

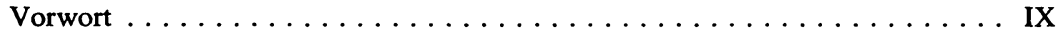

Einleitung - "Die Einzelnheiten sind eigentlich das Leben" $\ldots \ldots \ldots \ldots \ldots 1$

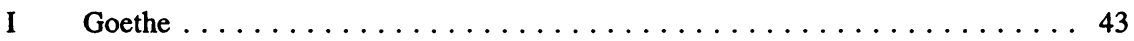

1 Lyrik - Die drei Zyklen der "Xenien", "Sonette" und des "West-östlichen

Divan" als Stadien der produktiven Rezeption ............ 43

$1.1 \quad$ Die "Xenien" als Wendepunkt .............. 43

$1.2 \quad$ Zelters Privileg: die Exklusivität des Sonettenzyklus von $1808 \ldots 53$

1.3 Der "West-östliche Divan" als Gipfelpunkt der Koproduktion und sprachschöpferischen Herausforderung $\ldots \ldots \ldots \ldots \ldots 61$

1.3.1 Der Sommer 1814 als Periode der engsten Zusammenarbeit von Goethe und Zelter . . . . . . . . . . . . . . 65

1.3.2 Das "gesellige Lied" Goethes und Zelters und die Entstehung der frühen Divan-Gedichte . . . . . . . . 69

1.3.3 Die Diskussion des "West-östlichen Divans" im Briefwechsel ..................... 90

2 Dramen - Goethes "Auge und Ohr in Berlin" : Zelters Theaterberichte und die Diskurse über dramatische Literatur $\ldots \ldots \ldots \ldots \ldots \ldots 100$

2.1 "zum Auge werden" - Goethes Dramen und ihre Realisierung auf der Bühne . . . . . . . . . . . . . . . . . . . . . 100

2.1.1 Die frühen Dramen $\ldots \ldots \ldots \ldots \ldots \ldots \ldots$

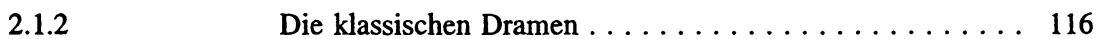

2.1.3 "Faust" $\ldots \ldots \ldots \ldots \ldots \ldots \ldots \ldots \ldots \ldots \ldots \ldots \ldots$

2.1.4 Festspiele $\ldots \ldots \ldots \ldots \ldots \ldots \ldots \ldots \ldots \ldots$ 
"Das Marterwesen und die heillosen Quälodramen" - Das Gegenbild des populären zeitgenössischen Dramas ........

Epik - Zelters Erweckungserlebnis und die Früchte "intensiver Lektüre"

Autobiographik - "das bekannte Bierschild einer pure vérité": Gestalteter Lebensweg, imaginäre und erlebte Reisen - Die Diskurse über Voraussetzungen, Formen und Inhalte der Autobiographik . . . . . . . .

"wird das Ding zum Roman werden müssen, um wahr zu erscheinen" - Zelters Autobiographie und "Dichtung und Wahrheit" oder über höhere Wahrheit und die Vorzüge "poetischer Geschichtsschreibung" . . . . . . . . . . . . . . . . . . . . 207

"ich bin mit Dir, bei Dir, ich bin in Rom" - Ein Höchstmaß an Vergegenwärtigung, Illusion und Identifikation im direkten Nachvollzug der "Italienischen Reise" . . . . . . . . . . . 215

"dem guten Prediger zu der Ehre verhelfen, etwas Gescheutes gemeint zu haben" - Das "Sanct Rochus-Fest zu Bingen" ... . . 233

Zur "Erweiterung der Grenzen des Klassischen" und ihren Grenzen

- Die Diskurse zur Kunst und zu Goethes Schriften zur Kunst . . 236

Die klassische Schule des Sehens - "Meles und Kritheis" als Gegenbild zu Judas Ischariot

Ein schwacher Nachklang der Auseinandersetzung um klassische und romantische Kunst - Der Diskurs über das Zelter-Porträt von Begas ............... 243

"Die Samariterin" von Wilhelm Hensel und "Cephalus und Prokris nach Julius Roman" . . . . . . . . . . . . . . . . . . . 247

"Gegenstände alter Kunst (...) wiederherzustellen" "Myrons Kuh" und "Philostrats Gemälde" . . . . . . . . 250 
"so nimm es nur symbolisch" / "Analog Denkende verstehen sich" - Einvernehmliche Grundlagen und Ziele von Zelters Rezeption der naturwissenschaftlichen Schriften Goethes . . . . . . . . 257

II Schiller und die deutsche Literatur neben Goethe . . . . . . . . . . 273

$1 \quad$ Einflüsse der Schiller-Rezeption Zelters auf den eigenen Briefwechsel und die "Schatten im Gemälde" der "klassischen" Freundschaft . . . . . 273

1.1 Zum Verhältnis von Zelter und Schiller nach externen Quellen . . 273

1.2 Die Rezeption des "Briefwechsels zwischen Schiller und Goethe" und die Auswirkungen des Gegenbildes auf den eigenen Briefwechsel ......................... 278

1.3 Die Diskurse zu Schillers Dramen im "Briefwechsel zwischen

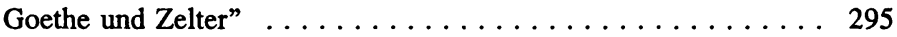

2 Lessing, Klopstock, Wieland, Herder: Beiträge zu einer Literaturgeschichte des 18. Jahrhunderts $\ldots \ldots \ldots \ldots \ldots \ldots \ldots \ldots$

3 Polemik und eine Poetologie der Vermeidung und des Verschweigens: Auseinandersetzungen mit der Literatur der Romantik . . . . . . . 335

III Weltliteratur $\ldots \ldots \ldots \ldots \ldots \ldots \ldots \ldots \ldots \ldots \ldots \ldots \ldots \ldots \ldots$

1 “die Vergleichungen lassen wir weg" - Die Behauptung des ÜberzeitlichKlassischen statt philologischer und historischer Kritik im Diskurs über antike Literatur $\ldots \ldots \ldots \ldots \ldots \ldots \ldots \ldots \ldots \ldots \ldots \ldots \ldots \ldots \ldots$

$1.1 \quad$ Griechische Literatur $\ldots \ldots \ldots \ldots \ldots \ldots \ldots \ldots \ldots$

$1.2 \quad$ Römische Literatur $\ldots \ldots \ldots \ldots \ldots \ldots \ldots \ldots \ldots \ldots$

2 "in Sprache, Form der Teile und des Ganzen unwidersprechlich, bequem

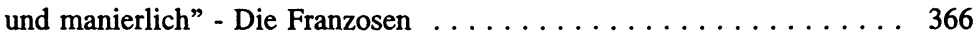

$2.1 \quad$ Das französische Theater $\ldots \ldots \ldots \ldots \ldots \ldots \ldots$

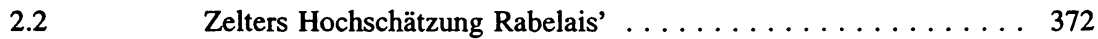

$2.3 \quad$ Die Literatur des 18. Jahrhunderts . . . . . . . . . . . . . . 374 
$3 \quad$ Englische Literatur . . . . . . . . . . . . . . . . . 390

Shakespeare - Reflexe lebenslänglicher Faszination und Ausein-

"Das Werk sei, wie es wolle, ich bin ihm Dank schuldig ... " Walter Scotts "Leben Napoleons" und die "subjektive" Lektüre

Bibliographie 


\section{Vorwort}

Die vorliegende Arbeit hat der Philosophischen Fakultät der Universität Münster vorgelegen und wurde im April 1997 als Dissertation angenommen. Für den Druck wurde sie geringfügig überarbeitet. Die Dissertation enthielt als Anhang die Rekonstruktion der "Bibliotheca Zelteriana", die jetzt als gesonderter Band in derselben Reihe erscheint.

Meinem Doktorvater, Herrn Prof. Dr. Hans Geulen, der das Thema anregte, bin ich für seine Betreuung und sein Interesse an meiner Arbeit zu besonderem Dank verpflichtet. Danken möchte ich auch dem Korreferenten, Herrn Prof. Dr. Ernst Ribbat.

Für zahlreiche wertvolle Auskünfte und Hinweise zu Zelter danke ich ganz herzlich Herm Prof. Dr. Peter Boerner (Bloomington, Indiana) und Frau Dr. Edith Zehm (München).

Hervorheben möchte ich auch die besondere Unterstützung, die mir von der Stiftung Weimarer Klassik (Herr Prof. Dr. Lothar Ehrlich, Weimar) und der Goethe-Gesellschaft (Herr Prof. Dr. Werner Keller, Köln) zuteil wurde, nicht zuletzt durch ein dreimonatiges Forschungsstipendium für meine weitergehenden Forschungen zu Zelters Korrespondenz, von der auch die vorliegende Arbeit profitieren konnte.

Alle Institutionen, die ich für meine Forschungen konsultiert habe, stellten mir ihre Bestände bereitwillig zur Verfügung und gaben mir auf meine Anfragen geduldig Auskunft. Besonderen Dank schulde ich in diesem Zusammenhang dem Goethe- und Schiller-Archiv in Weimar (Herrn Dr. Jochen Golz), der Sammlung Kippenberg/Goethe-Museum Düsseldorf (Herm Prof. Dr. Volkmar Hansen und Frau Regine Zeller) und der Staatsbibliothek Preußischer Kulturbesitz zu Berlin.

Abschließend möchte ich mich ganz herzlich bei meinen Eltern bedanken, die mich in meinen Interessen immer unterstützten und mir dieses Studium ermöglichten. Ihnen sei diese Arbeit gewidmet.

München, im August 1999 